J. Dairy Sci. 99:9383-9394

http://dx.doi.org/10.3168/jds.2016-10930

(C) American Dairy Science Association ${ }^{\circledR}, 2016$.

\title{
Goat whey ameliorates intestinal inflammation on acetic acid-induced colitis in rats
}

\author{
Daline Fernandes de Souza Araújo, ${ }^{*}$ Gerlane Coelho Bernardo Guerra, $\dagger^{1}$ \\ Raimundo Fernandes de Araújo Júnior,ł Aurigena Antunes de Araújo,† Paloma Oliveira Antonino de Assis,§ \\ Ariosvaldo Nunes de Medeiros,\# Yasmim Regis Formiga de Sousa, ${ }^{*}$ Maria Manuela Estevez Pintado,ll \\ Julio Gálvez, đ and Rita de Cássia Ramos do Egypto Queiroga§ \\ *Department of Science and Food Technology, Federal University of Paraíba, 58051-900 Santa Rita, Brazil \\ †Department of Biophysics and Pharmacology, and \\ ‡Department of Morphology, Histology and Basic Pathology, Biosciences Center, Federal University of Rio Grande do Norte, \\ 59078-970 Natal, Brazil \\ $\S$ Department of Nutrition, Health Sciences Center, and \\ \#Department of Animal Science, Federal University of Paraíba, 58051-900 Santa Rita, Brazil \\ IISchool of Biotechnology, Catholic University of Portugal, 4202-401 Porto, Portugal \\ ๆCentro de Investigación Biomédica en Enfermedades Hepáticas y Digestivas (CIBER-EHD), Instituto de Investigación Biosanitaria de Granada \\ (ibs.Granada), Center for Biomedical Research (CIBM), University of Granada, 18071 Granada, Spain
}

\begin{abstract}
Complementary or alternative medicine is of great interest for the treatment of inflammatory bowel disease, with the aim of ameliorating the side effects of the drugs commonly used or improving their efficacy. In this study, we evaluated the ability of goat whey to prevent intestinal inflammation in the experimental model of acetic acid-induced rats and compared it to sulfasalazine. Pretreatment with goat whey $(1,2$, and 4 $\mathrm{g} / \mathrm{kg})$ and sulfasalazine $(250 \mathrm{mg} / \mathrm{kg})$ on colitic rats improved colonic inflammatory markers, including myeloperoxidase activity, leukotriene $\mathrm{B}_{4}$ levels, as well as the production of proinflammatory cytokines IL- $1 \beta$ and tumor necrosis factor- $\alpha$. Furthermore, the administration of goat whey significantly reduced the colonic oxidative stress by reducing malondialdehyde levels and increased total glutathione content, a potent antioxidant peptide. The histological evaluation of the colonic specimens from colitic rats confirmed these beneficial effects, as goat whey preserved the colonic tissue, especially in those rats treated with the highest dose of goat whey or with sulfasalazine. The immunohistochemistry analysis of the colonic tissue evaluation also revealed a reduction in the expression of cyclooxygenase-2, inducible nitric oxide synthase, and matrix metalloproteinase-9, together with an increased expression of suppressor of cytokine signaling-1. These results suggest that goat
\end{abstract}

Received January 21, 2016.

Accepted June 17, 2016.

${ }^{1}$ Corresponding author: gerlaneguerra@hotmail.com whey exerted a preventive effect against the intestinal damage induced by acetic acid, showing a similar efficacy to that shown by sulfasalazine, therefore making it a potential treatment for human inflammatory bowel disease.

Key words: goat whey, intestinal inflammation, oxidative stress, cytokines, immunohistochemical

\section{INTRODUCTION}

Inflammatory bowel disease (IBD) is a chronic inflammatory condition, encompassing Crohn's disease and ulcerative colitis, with high incidence and prevalence in developed countries. The pathologies are characterized by pain, diarrhea, weight loss, frequent rectal bleeding, and alternating periods of relapse and remission. Both forms of IBD feature exacerbated uncontrolled intestinal inflammation that leads to poor quality of life and requires prolonged medical or surgical interventions (Molodecky et al., 2012; Nørgård et al., 2014). At present, the exact pathogenesis of IBD is not fully understood, as it is a complex and multifactorial chronic disease. Currently, there is general agreement that IBD is the result of the combined effects of 4 basic components: multiple genetic variations of the host, alterations in the composition of the intestinal microbiota, changes in the surrounding environment that are greatly affected by diet, and the abnormal reactivity of the intestinal mucosal immune response to an unknown antigen (Galvez et al., 2010; Sang et al., 2014; Magalhães et al., 2015).

Different studies have proposed that this inflammatory process is initially triggered by the increased permeability of the epithelial barrier to the luminal 
antigens, thus leading to an improper activation of the immune mucosal system. This results in an increased influx and activation of neutrophils and macrophages, with the concomitant production of several proinflammatory mediators, including reactive oxygen species, eicosanoids, cytokines, and chemokines (Dedon and Tannenbaum, 2004; Hyun and Mayer, 2006; Strober and Fuss, 2011). Moreover, colitis has been associated with the increased expression of inducible proteins such as cyclooxygenase (COX)-2 and inducible nitric oxide synthase (iNOS), which may play a key role in the modulation of inflammation (Ardizzone and Porro, 2005; Sakthivel and Guruvayoorappan, 2013). In fact, the synthesis of large amounts of nitric oxide by iNOS has been demonstrated to be pathogenic in human IBD (Kolios et al., 2004). Of note, it has been reported that the increased expression of iNOS is mediated through the activation of the transcription factor nuclear factor (NF) $-\kappa B$ and driven by different proinflammatory cytokines, such as tumor necrosis factor (TNF)- $\alpha$, IL-1 $\beta$ and IFN- $\gamma$. Subsequently, NF- $\kappa \mathrm{B}$ activation can also induce the expression of these cytokines, thus generating a vicious cycle, as well as that of many other genes involved in antiapoptotic and prometastatic processes such as matrix metalloproteinase (MMP) 2 and 9, respectively (Salas et al., 2002; Agarwal et al., 2004; Naugler and Karin, 2008; Rouzer and Marnett, 2009).

Treatment of IBD has 2 main purposes: to promote the remission of the symptoms of the acute period and then to maintain the remission by controlling the chronic inflammation, thus preventing the reactivation of the intestinal inflammatory process. When considering all of the above, the downregulation of the altered immune response is essential for the management of these patients. In fact, this is the main objective of the pharmacological therapy at present, which includes aminosalicylates (sulfasalazine or mesalazine), immunosuppressants (glucocorticoids or azathioprine), and biologicals (infliximab or adalimumab; Bernstein, 2015). However, although all these drugs have shown efficacy, an important proportion of side effects may limit their required long-term use (Siegel, 2011). In this context, new therapies that combine efficacy and safety in human IBD therapy are needed. Among these, dietary interventions with nutraceuticals or so-called functional foods seem to represent a safe alternative way to modulate the altered mucosal immune response that occurs in intestinal inflammation, which can be achieved mainly through their effect on intestinal microbiota, as has been reported for prebiotics (Roberfroid et al., 2010).

Whey is a by-product of cheese and contains nutritionally important and valuable components used in a functional food. In fact, whey is already considered as a functional milk fraction, with positive effects on health outcomes, as it contains proteins with a high nutritional value due to the presence of EAA, as well as considerable lactose content, oligosaccharides, and minerals (Hernández-Ledesma et al., 2011; Thum et al., 2015). The aim of our study was to evaluate the preventive effects of goat whey $(\mathbf{G W})$ in an experimental model of intestinal inflammation induced by intracolonic instillation of acid acetic in rats, and compare its efficacy to sulfasalazine (SAZ), an aminosalicylate currently used in human IBD.

\section{MATERIALS AND METHODS}

\section{Obtaining and Characterizing the GW}

Goat cheese whey came from alpine brown goats confined at the experimental station of the Goat Division of the Federal University of Paraiba, in São João do Cariri, Paraiba, Brazil. The selected subjects weighed $40 \pm 6 \mathrm{~kg}$ of BW and were $50 \pm 10 \mathrm{~d}$ of lactation and had received full ration consisting of concentrate, Tifton hay, and forage cactus. The diets were formulated according to NRC (2007) to meet the required milk production of $1.5 \mathrm{~kg}$ per day with $4 \%$ fat.

Next, we proceeded to prepare the cheese curds and obtain whey (Oliveira et al., 2012). Initially, milk pasteurization $\left(65 \pm 1^{\circ} \mathrm{C}\right)$ was performed for $15 \mathrm{~min}$ followed by cooling to $45 \pm 1^{\circ} \mathrm{C}$. Then, $\mathrm{GW}$ was produced by coagulation with additives in the following sequence: $0.5 \mathrm{~mL} / \mathrm{L}$ of calcium chloride and $0.9 \mathrm{~mL} / \mathrm{L}$ of commercial coagulant chloride (Ha-La, Chr. Hansen A/S, Hørsholm, Denmark). The mass was salted ( $\mathrm{NaCl} 1 \%$ relative to the weight of the mass) and distributed in a 250 -g perforated form.

The whey obtained from the preparation of the cheese curd was immediately stored in polyethylene containers and subjected to spray-drying by a mini $\mathrm{Bu}-$ chi B290 Spray Dryer (Büchi Labortechnik AG, Flawil, Switzerland), using an inlet temperature of $130^{\circ} \mathrm{C}$, feed rate of $15 \%$, air flow of $40 \mathrm{~L} / \mathrm{min}$, and a compressor pressure of around $700 \mathrm{kPa}$ for an approximate time of $2 \mathrm{~h}$ drying for $1 \mathrm{~L}$ of whey. Analyses were performed to characterize the GW to understand the moisture (DM basis), fat (Gerber butyrometer, Gerber Instruments, Effretikon, Zürich, Switzerland), protein (by the microKjeldahl method), and lactose (by HPLC, Varian, Palo Alto, CA); all analyses were performed according to the methods described by the AOAC International (2005). The preparation of sialic acid whey solution and quantification were carried out following the methodology used by Sousa et al. (2015). 


\section{Chemicals and Reagents}

The standards for the identification and quantification of $N$-acetylneuraminic acid of sialic acid (NeuAc/ Z98\%), $\mathrm{N}$-glycolylneuraminic acid (NeuGc/Z95\%), 1,2-diamino-4,5-methyleneoxybennzene derivatization reagent, and the reagents used for the determination of total glutathione content, myeloperoxidase (MPO) activity, and malondialdehyde (MDA) levels were purchased from Sigma Aldrich (São Paulo, SP, Brazil). Rat ELISA kits [IL-1 $\beta$, TNF- $\alpha$, and leukotriene $\mathrm{B}_{4}\left(\mathbf{L T B}_{4}\right)$ ] and the polyclonal antibodies COX-2, iNOS, suppressor of cytokine signaling-1 (SOCS-1), and MMP 9 were purchased from R\&D Systems (Minneapolis, MN) and Santa Cruz Biotechnology Inc. (Dallas, TX), respectively. Streptavidin-HRP-conjugated secondary antibody and TrekAvidin-HRP Label + Kit were from Biocare Medical (Concrod, CA). Other chemicals used were of the highest grade available.

\section{Ethics Statement}

This study was approved by the Ethics Committee on Animal Use (protocol no. 0608/2013) from Federal University of Paraiba. The animal care and research protocols were based on the principles and guidelines adopted by the Guide for the Care and Use of Laboratory Animals (NRC, 1985).

\section{Experimental Design}

Experiments were performed on female Wistar rats $(220 \pm 20 \mathrm{~g}$ of BW), obtained from the Biotechnology Center/Universidade Federal da Paraiba, and housed in standard conditions (12-h light/dark cycle, $22 \pm 0.1^{\circ} \mathrm{C}$, and $50-55 \%$ humidity) with ad libitum access to food (Presence diet) and water.

The animals were divided into groups $(\mathrm{n}=10$ per group): acetic acid control, colitic groups treated with different doses of $\mathrm{GW}(1,2$, or $4 \mathrm{~g} / \mathrm{kg})$ or SAZ (250 $\mathrm{mg} / \mathrm{kg}$ ), and noncolitic. The test compounds were suspended in $1 \mathrm{~mL}$ of water and orally administered by gavage. All treatments were started $14 \mathrm{~d}$ before colitis induction and continued until euthanizing the rats, which took place $72 \mathrm{~h}$ after. The control and noncolitic group only received the vehicle orally (1 $\mathrm{mL}$ of water).

\section{Acetic Acid-Induced Colitis}

The animals were fasted overnight and anesthetized with ketamine $(70 \mathrm{mg} / \mathrm{kg}, 10 \%)$ and xylazine $(10 \mathrm{mg} /$ $\mathrm{kg}, 2 \%$ ). Colitis was induced according to methods originally described by MacPherson and Pfeiffer (1978) and modified by Millar et al. (1996). The catheter was then carefully inserted into the colon and the tip was $8 \mathrm{~cm}$ proximal to the anus of the rats. Then, $10 \%$ acetic acid $(0.5 \mathrm{~mL})$ in $0.9 \%$ saline was instilled into the lumen of the colon. The noncolitic group received $0.5 \mathrm{~mL}$ of saline $0.9 \%$ intracolonically. The rats were maintained in a supine Trendelenburg position for $30 \mathrm{~s}$ to prevent the leakage of the intracolonic instillation.

\section{Disease Activity Index}

The disease activity index (DAI) was used for evaluating the grade and extent of intestinal inflammation, which was quantified with a clinical score assessing weight loss, stool consistency, and gross bleeding divided by 3 . We determined scores for change in $\mathrm{BW}$ loss $(0=$ none, $1=1-5 \%, 2=5-10 \%, 3=10-20 \%, 4=$ $>20 \%)$, stool blood $(0=$ negative, $1=+, 2=++, 3=$ $+++, 4=++++)$, and stool consistency $(0=$ normal, 1 and $2=$ loose stool, 3 and $4=$ diarrhea).

\section{Assessment of Colonic Damage}

The animals were fasted overnight and euthanized with an overdose of thiopental; then, the colon was removed aseptically, rinsed with PBS, and placed on an ice-cold plate. The colon was cleaned of fat and mesentery. Then, each specimen was weighed and its length measured under a constant load (2 g). Afterward, the colon was longitudinally opened and scored for macroscopically visible damage on a 0-to-10 scale according to the criteria described by Bell et al., (1995). The colon was subsequently longitudinally divided into segments for biochemical determinations, which were performed in all the samples. Colonic fragments were frozen at $-80^{\circ} \mathrm{C}$ for biochemical evaluation.

\section{Biochemical Evaluation of Colonic Damage}

The MPO activity was measured according to the technique described by Krawisz et al. (1984). Results were calculated as the quantity of enzyme degrading 1 $\mu \mathrm{mol} / \mathrm{mL}$ of peroxide at $25^{\circ} \mathrm{C}$ and expressed in units per milligram of tissue. Total glutathione content was quantified with the recycling assay described by Anderson (1985), and tissue MDA levels, an index of lipid peroxidation, were determined following the methodology described by Esterbauer and Cheeseman (1990), which is based on the reaction of a chromogenic reagent (1-methyl-2-phenylindole). The results from total glutathione content and MDA were expressed as nanomoles per gram of wet tissue and the absorbance of MPO activity, total glutathione, and MDA were measured with absorbance at 450, 412, and $586 \mathrm{~nm}$, respectively. 


\section{Cytokines and $\mathrm{LTB}_{4}$ Levels}

The rat cytokines IL-1 $\beta$, TNF- $\alpha$, and the eicosanoid $\mathrm{LTB}_{4}$ were determined in colon homogenate supernatants using commercial ELISA kits (R\&D Systems, Minneapolis, MN). Next, the plates were coated overnight with capture antibodies against rat. After, the plates were washed 3 times with buffer (repeated between each step with the substrate solution), and then they were blocked with Reagent Diluent-RD (R\&D Systems). The standards at various dilutions and samples were added in duplicate and incubated at $37^{\circ} \mathrm{C}$ for $2 \mathrm{~h}$. Then, biotinylated sheep polyclonal antibodies (anti-IL-1 $\beta$, anti-TNF- $\alpha$, or anti-LTB ${ }_{4}$ ) were added to the wells. After incubation at room temperature for 1 $\mathrm{h}$, plates were washed and $100 \mu \mathrm{L}$ of streptavidin-HRPconjugated were added. Solutions A and B of the commercial ELISA kit were added for $20 \mathrm{~min}$. The enzyme reaction was stopped with $\mathrm{H}_{2} \mathrm{SO}_{4}(2 N)$ stop solution and absorbance was measured at $450 \mathrm{~nm}$. Results were expressed as cytokine concentration $(\mathrm{pg} / \mathrm{mL})$.

\section{Histopathology and Immunohistochemical Analysis}

Colon samples were collected and fixed in buffered paraformaldehyde (10\% in PBS, pH 7.2) for $24 \mathrm{~h}$. Crosssections were selected and embedded in paraffin. Three tissue sections $(5 \mu \mathrm{m})$ of 5 animals per group were obtained with a microtome and stained with hematoxylin and eosin for histological evaluation by optical microscopy. Histological sections were also evaluated by the degree of leukocyte infiltration and distribution, using the parameters described by Zea-Iriarte et al. (1996); reported histopathological analyses were performed independently by 2 pathologists, blinded to the group identity.

Thin colon sections $(3 \mu \mathrm{m})$ of 5 per group were obtained with a microtome and transferred to gelatincoated slides. Each tissue section was then deparaffinized and rehydrated. Intestinal tissue slices were washed with $0.3 \%$ Triton X-100 (Sigma-Aldrich, São Paulo, Brazil) in phosphate buffer, wet with endogenous peroxidase ( $3 \%$ hydrogen peroxide), and incubated overnight at $4^{\circ} \mathrm{C}$ with the following primary antibodies: COX-2, 1:500; iNOS, 1:500; MMP-9, 1:800; SOCS-1, 1:800. The analyses conducted were similar to those performed by Guerra et al. (2015).

\section{Statistical Analysis}

All results were expressed as means \pm standard error of the means. Differences between means were tested for statistical significance using one-way ANOVA followed by Tukey test. Nonparametric data (score) are expressed as the median (range) and were analyzed using the Mann-Whitney test. All statistical analyzes were performed using GraphPad 5.0 software (GraphPad Software Inc., La Jolla, CA) and statistical significance was set at $P<0.05$.

\section{RESULTS}

The characterization of GW revealed the presence of humidity on dry base $(12.16 \pm 0.69 \mathrm{~g} / 100 \mathrm{~g})$, proteins $(5.96 \pm 0.30 \mathrm{~g} / 100 \mathrm{~g})$, fat $(2.56 \pm 0.25 \mathrm{~g} / 100 \mathrm{~g})$, lactose $(15.62 \pm 1.08 \mathrm{~g} / 100 \mathrm{~g})$, and sialic acid $(66.63 \pm 2.34$ $\mathrm{mg} / \mathrm{L}$ ) as the sum of $N$-acetylneuraminic acid of sialic acid $(24.91 \pm 1.54 \mathrm{mg} / \mathrm{L})$ and $N$-glycolylneuraminic acid $(41.72 \pm 0.80 \mathrm{mg} / \mathrm{L})$.

The administration of GW or SAZ to rats for $14 \mathrm{~d}$ before colitis induction did not have any deleterious effects on BW (Figure 1A) or food and water intake when compared with the untreated groups (data not shown). Once colitis was induced, treated groups showed a lower effect of the intestinal inflammatory process, with lower BW loss as well as a decrease in the occurrence of diarrhea and presence of fecal blood. This resulted in improvement in the evolution of the DAI values in comparison with the colitic control group, which was statistically different $(P<0.05)$ in those groups receiving SAZ and the highest doses of whey (Figure 1B).

Once the rats were euthanized, the macroscopic evaluation of the colonic specimens revealed that the intrarectal administration of acetic acid caused intense damage in the large intestine. This was characterized in the colitic control group by the presence of gross ulceration and inflammation areas, typically affecting 4 to $8 \mathrm{~cm}$ of the colon, with a score of 7 to 10 . The treatment of the colitic rats with GW showed a reduced effect $(P<0.05)$ of the inflammatory process, as evidenced by the lower colonic macroscopic score values obtained, which achieved statistical differences $(P<0.05)$ at doses of 2 and $4 \mathrm{~g} / \mathrm{kg}$ in comparison with the control group, and showed a similar efficacy to that of SAZ (Figure 1C). These beneficial effects were associated with a reduced weight-to-length ratio of the colonic segment $(P<0.05)$ in comparison to the colitic control group (Figure 1D). This reflected an improvement in the tissue edema and thickening associated with the colonic inflammatory process together with the shortening of the rat's large intestine.

The histological evaluation of the colonic specimens confirmed the intestinal anti-inflammatory effects exerted by either GW, especially at doses of 2 and $4 \mathrm{~g} /$ $\mathrm{kg}$ (Figure 2E and 2F, respectively), or SAZ (Figure 2C). When compared with normal colonic architecture, the colitic control group (Figure 2B) showed signs of intense transmural inflammation, involving both the 
A

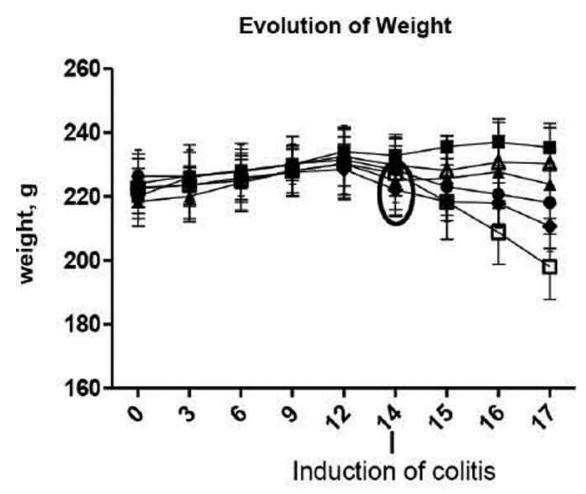

B

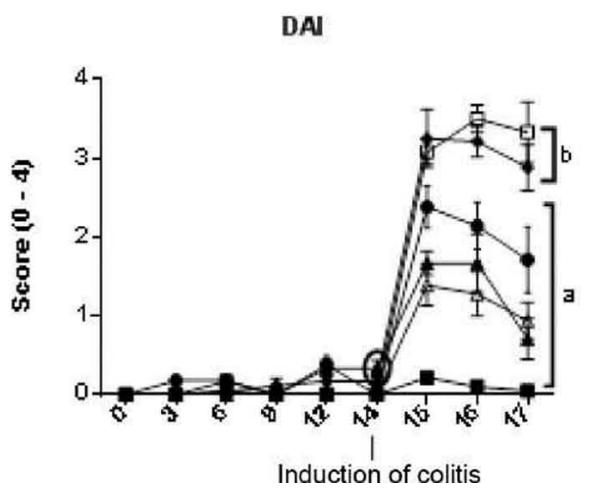

C

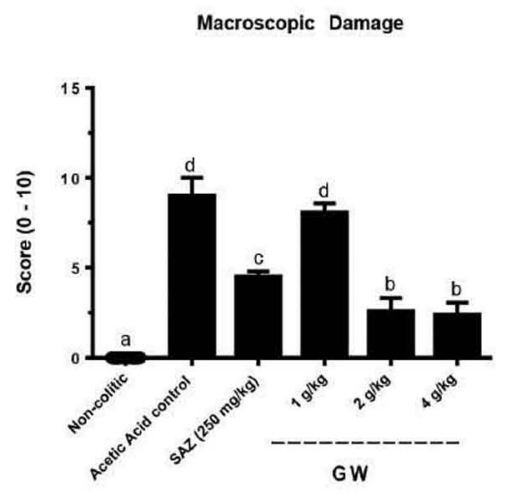

D

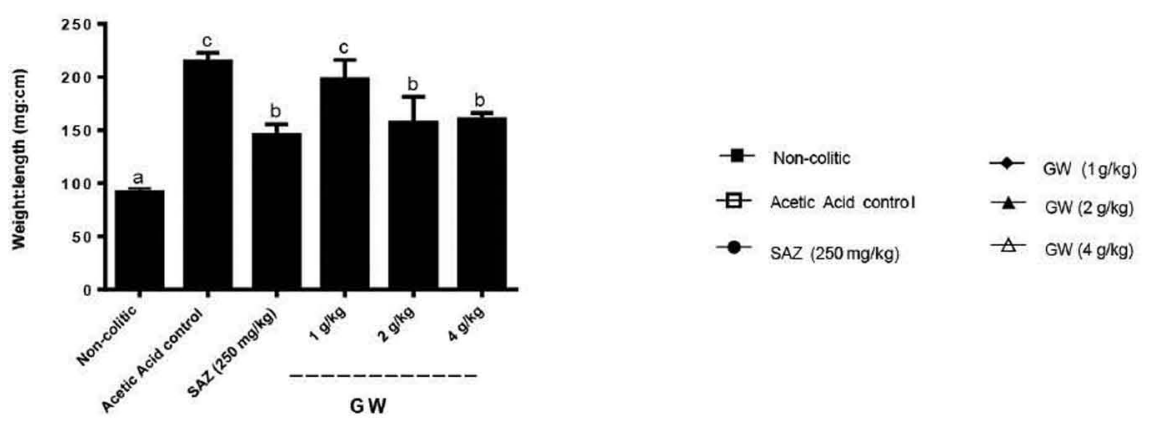

Figure 1. Effect of treatments of goat whey (GW) and sulfasalazine (SAZ) on evolution of weight (A), disease activity index (DAI; B), macroscopic damage score (C), and weight-to-length ratio (D) in acetic acid-induced intestinal inflammation (colitis) in rats. Panel E shows a representative photograph of intestine from each experimental group. Data $(\mathrm{n}=10)$ are presented as means \pm SEM. Groups with different letters $(\mathrm{a}-\mathrm{d})$ statistically differ $(P<0.05)$. Color version available online.

mucosa and submucosa layers. Extensive ulceration was also noted, which affected most of the colonic surface and resulted in almost complete destruction of crypts and the absence of goblet cells. The inflammatory process was also associated with severe leukocyte infiltration in all the intestine layers. The colonic tissue from colitic rats treated with SAZ or GW (2 and $4 \mathrm{~g} /$ $\mathrm{kg}$ ) showed a partial recovery of the mucosa and submucosa, with a lower inflammatory infiltrate, together with the emerging presence of crypts and goblet cells. The beneficial effect of these treatments on colonic architecture was associated with a significantly lower $(P$ $<0.05)$ histopathological score in comparison with the colitic control group.

Moreover, the intestinal anti-inflammatory effect was also biochemically confirmed by an improvement in most of the assayed markers. In this regard, colonic inflammation was associated with increased MPO activity (Figure 3A), thus reflecting the increased neu- trophil infiltration that characterizes this inflammatory process, thereby also confirming the observations from the histological analysis. Treatment of colitic rats with GW or SAZ resulted in a significant decrease $(P<$ $0.05)$ in this enzyme activity, in accordance with the results obtained in the histological studies (Figure 2) in these groups of animals, in which a reduced leukocyte infiltration was observed. Additionally, when the levels of the chemotactic eicosanoid $\mathrm{LTB}_{4}$ (Figure 3B) were evaluated, it was observed that the inflammatory process was associated with an increased production in comparison with noncolitic rats, with these being significantly downregulated $(P<0.05)$ after treatment with either GW (2 and $4 \mathrm{~g} / \mathrm{kg}$ ) or SAZ.

In addition, it was observed that the colonic inflammatory was associated with oxidative stress, evidenced by a depletion of antioxidant peptide glutathione (Figure 3C) as well as by an increased production of colonic MDA (Figure 3D), a final product of lipid peroxidation. 
This colonic oxidative stress was significantly counteracted after treating the colitic rats with GW, 2 and 4 $\mathrm{g} / \mathrm{kg}(P<0.05)$, or with SAZ $(P<0.05)$.
Likewise, the colonic production of the proinflammatory cytokines IL-1 $\beta$ (Figure 4A) and TNF- $\alpha$ (Figure 4B) were significantly increased $(P<0.05)$ in the co-
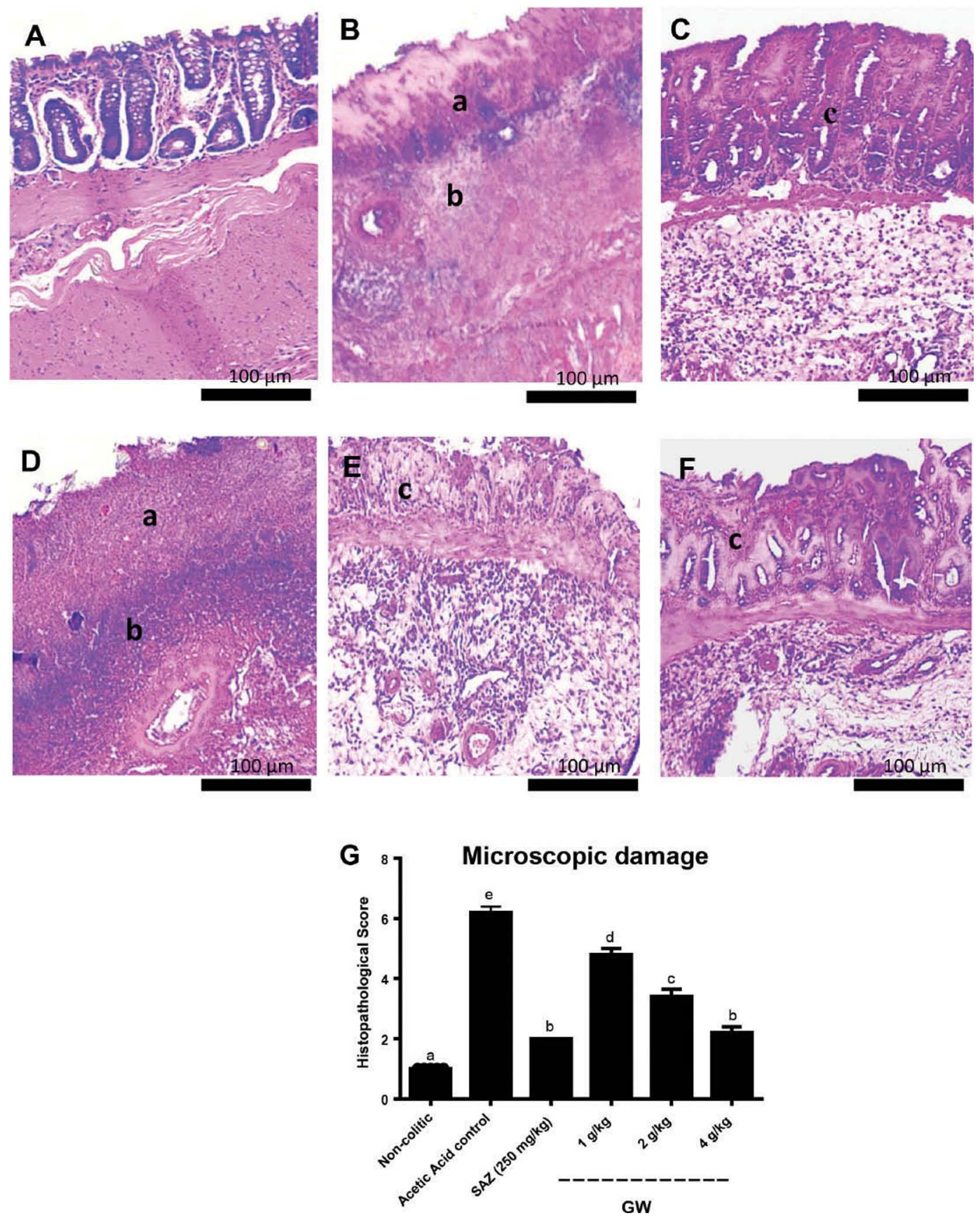

Figure 2. Histopathologic features of representative colonic tissue of rats, showing the colon fragment cut in the longitudinal direction. Normal intestinal layers (A). Intense (B) or moderate (D) inflammation in the (a) mucosa and (b) submucosa with infiltrate of neutrophils; (c) reduced inflammatory infiltrate, or integrated epithelial cells with slight goblet cell loss, fewer cells infiltrate and fibrous connective tissue hyperplasia in colonic mucous membrane were usually found $(\mathrm{C}, \mathrm{E}$, and $\mathrm{F} ; \times 100)$. SAZ = sulfasalazine; $\mathrm{GW}=$ goat whey; $\mathrm{A}=$ noncolitic; $\mathrm{B}=$ acid acetic control; $\mathrm{C}=$ sulfasalazine; $\mathrm{D}=1 \mathrm{~g} / \mathrm{kg}$ of $\mathrm{GW} ; \mathrm{E}=2 \mathrm{~g} / \mathrm{kg}$ of GW; F $=4 \mathrm{~g} / \mathrm{kg}$ of GW. Panel G shows histological scores for each treatment $(3$ sections/animal). Data $(\mathrm{n}=10)$ are expressed as means \pm SEM. Groups with different letters $(\mathrm{a}-\mathrm{e})$ statistically differ $(P<0.05)$. Color version available online. 
A

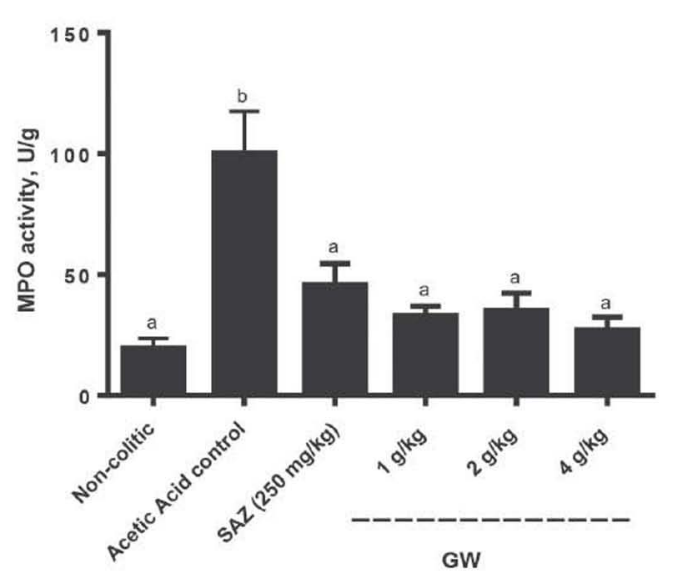

C

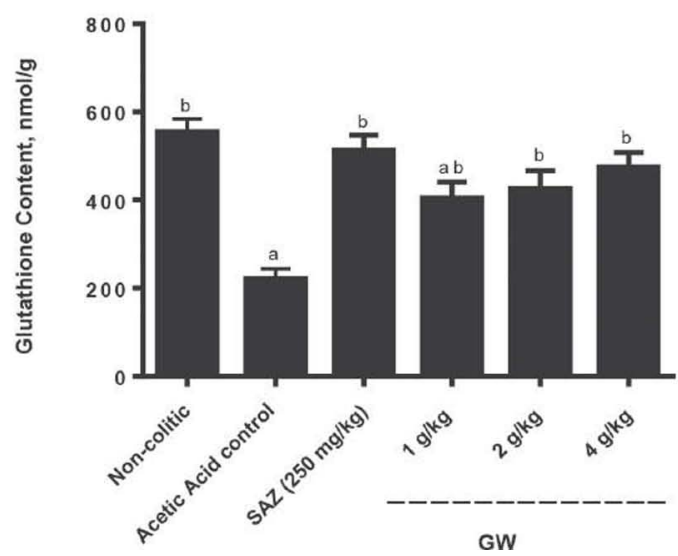

B

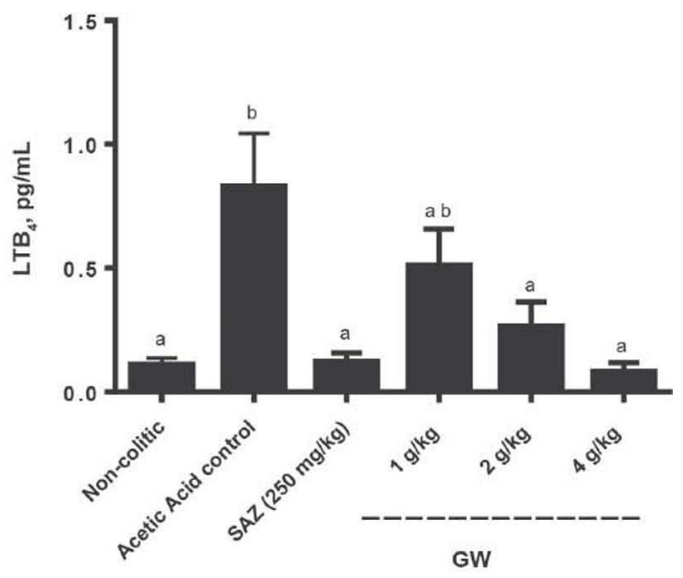

D

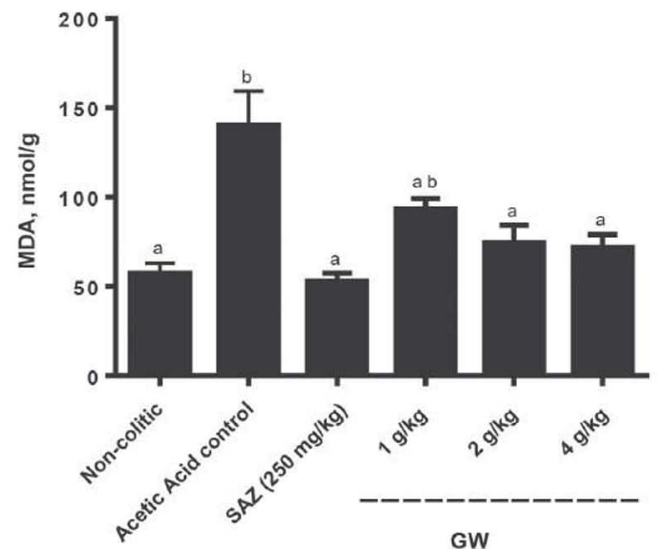

Figure 3. Effect of the goat whey $(\mathrm{GW})$ and sulfasalazine (SAZ) in the myeloperoxidase (MPO) activity (A), leukotriene $\mathrm{B}_{4}\left(\mathrm{LTB}_{4}\right.$; $\mathrm{B}_{\text {), }}$ glutathione content $(\mathrm{C})$, and malonaldehyde (MDA; D) levels in the intestinal inflammation of the experimental model of colitis in acid-induced rats. Data $(\mathrm{n}=10)$ are expressed as means \pm SEM. Groups with different letters $(\mathrm{a}, \mathrm{b})$ differ statistically $(P<0.05)$.

A

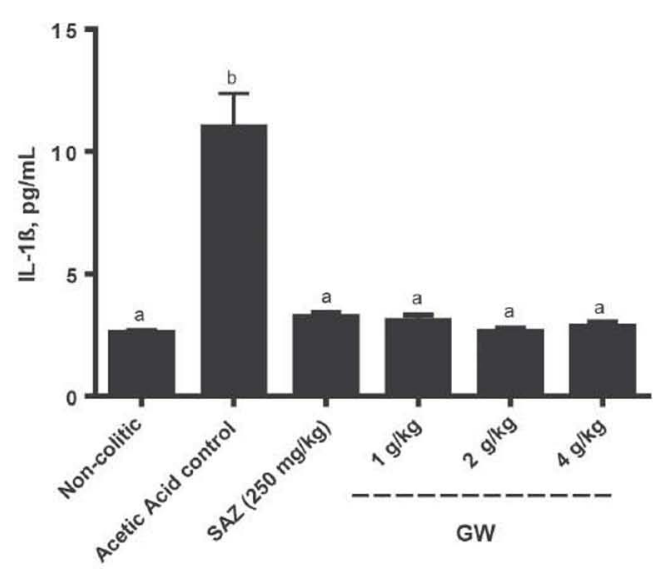

B

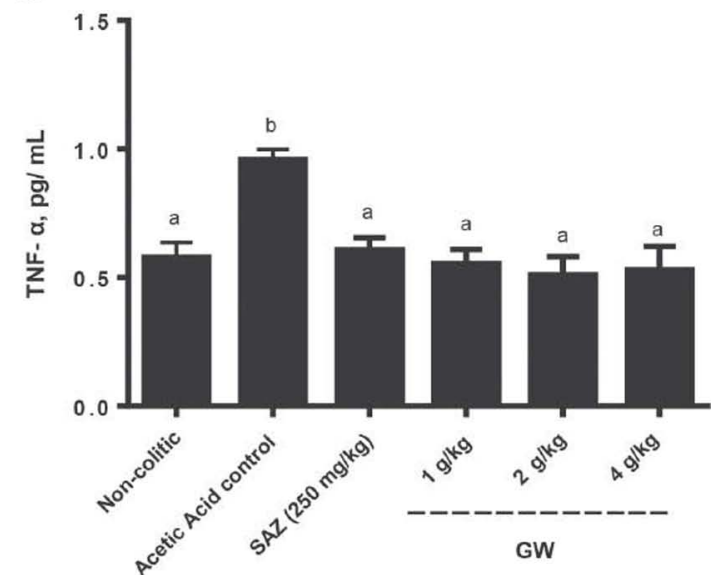

Figure 4. Effect of different doses of goat whey $(\mathrm{GW})$ and sulfasalazine (SAZ) in IL-1 $\beta$ and tumor necrosis factor (TNF)- $\alpha$ levels in the intestinal inflammation of the experimental model of colitis in acid-induced rats. Data $(\mathrm{n}=10)$ are expressed as means \pm SEM; groups with different letters $(\mathrm{a}, \mathrm{b})$ differ statistically $(P<0.05)$. 
lonic segments from control colitic rats in comparison with the healthy group. Once again, the treatment of colitic rats with GW, at all doses, or with SAZ resulted in a significant decrease compared with the acetic acid control group $(P<0.05)$.

To obtain additional information regarding the effects of the treatments on intestinal inflammation, immunohistochemical analysis were performed in those groups treated with SAZ or GW at the highest assayed dose. These studies revealed that acetic acid-induced intestinal inflammation was associated with a strong labeling for the inducible proteins COX-2, iNOS, and MMP-9 (Figure 5; 2A, 2B, and 2D), when compared with the noncolitic group (Figure 5; 1A, 1B, and 1D). However, the expressions for these enzymes were markedly reduced in those colitic rats that received the treatments SAZ (Figure 5; 3A, 3B, and 3D) and GW (Figure 5; 4A, 4B, and 4D), without evident differences between both groups. On the other hand, when the expression of SOCS-1 was evaluated, noncolitic rats (Figure 5; 1C) and both treated colitic groups (SAZ and GW) showed an increased expression (Figure 5; 3C and $4 \mathrm{C}$, respectively) in comparison with that observed in the corresponding control colitic group (Figure 5; $2 \mathrm{C}$ ), which showed a significant reduced expression of this protein.

\section{DISCUSSION}

Despite the progress made in recent years in the treatment of IBD, there is still a clear demand for new therapeutic strategies to manage these intestinal conditions. At present, the pharmacological treatment includes the use of drugs such as 5-aminosalicylic acid derivatives, including sulfasalazine, corticosteroids, immunosuppressants such as azathioprine or methotrexate, and biologicals such as anti-TNF therapy (Bernstein, 2015; Bressler et al., 2015). However, and unfortunately, some of these drugs may not have the desired effects on the patient or may even provoke side effects, especially when used for prolonged periods (Siegel, 2011). For this reason, we have noted increasing interest in the development of new strategies, including the use of alternative medicines or bioactive food components, which can collaborate with the standard drug treatments to restore the inflamed intestine, prevent the relapses that characterize these intestinal conditions, and reduce the risks of adverse effects (Hur et al., 2012).

In this sense, GW can be considered as an important source for dietary bioactive components, including peptides, proteins of high biological value, and oligosaccharides, as found in this study. It is interesting to note that the content of oligosaccharides has been reported to be higher in GW than that from other ruminants (Martinez-Ferez et al., 2006; Albrecht et al., 2014; Ahmed et al., 2015).

Thum et al. (2015) also found higher values for protein, fat, and lactose in GW, and about $0.20 \mathrm{~g} / \mathrm{L}$ and $60 \mathrm{mg} / \mathrm{L}$ of oligosaccharides and $\mathrm{N}$-glycolylneuraminic acid, respectively. The change in oligosaccharide concentrations can occur due to variation of the lactation period, food type, and even quantification method (Martinez-Ferez et al., 2006).

Oligosaccharides are glycans composed of lactose with branches or $N$-acetyl lactosamine, with sialic acid and fucose in their chains. Sialic acid reduces the adhesion of leukocytes on endothelial cells, presenting an indication of the immunoregulatory effects of human milk oligosaccharides (Kunz et al., 2000).

Previous studies have reported the intestinal anti-inflammatory properties of oligosaccharides isolated from goat milk in an experimental model of murine colitis (Daddaoua et al., 2006; Lara-Villoslada et al., 2006). Based on these observations, it is plausible that GW could exert beneficial effects on intestinal inflammation. In fact, the present study showed the preventive intestinal anti-inflammatory activity of GW in an experimental model of acetic acid-induced colitis in rats, showing a similar efficacy to SAZ $(250 \mathrm{mg} / \mathrm{kg}$ per day), a drug currently used for the treatment of human IBD. This was observed when the colonic specimens from colitic rats were evaluated macroscopically, microscopically, and biochemically.

In the course of the experiment, both GW and SAZ were able to decrease the DAI values in comparison with untreated control group. The DAI clearly correlates with the severity of the disease, thus reflecting the effect of the colonic inflammatory process on rat weight, stool consistency, and the presence of blood in the feces.

In IBD, the typical patients can display different gastrointestinal symptoms, including abdominal pain, diarrhea, and rectal bleeding, which can also be associated with other systemic symptoms such as weight loss, fever, and fatigue (Strober et al., 2007). In the preclinical trial, DAI was clearly correlated with the severity of disease; thus, the improvement of this parameter reflects the GW potential in the treatment of IBD.

The improvement of the inflammatory process after treatment with $\mathrm{GW}$ is a consequence of the positive effects on colonic inflammation, as revealed by the increase in rat BW, as well as after macroscopic evaluation of the colonic segments showing a reduction in macroscopic score and an improvement of weight-tolength ratio. In addition, the positive effects by the treatments were also seen histologically, as a recovery of the inflammatory process in association with a re- 
duction in the inflammatory neutrophil infiltrate was noted after microscopic evaluation of the colonic specimens in comparison with colitic rats.
The lower neutrophil infiltration in the colonic tissue was biochemically confirmed when colonic MPO activity was evaluated. This can clearly contribute to the

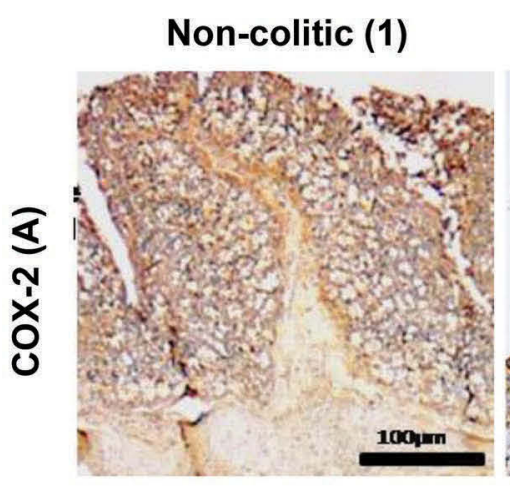

Acetic Acid Control (2)
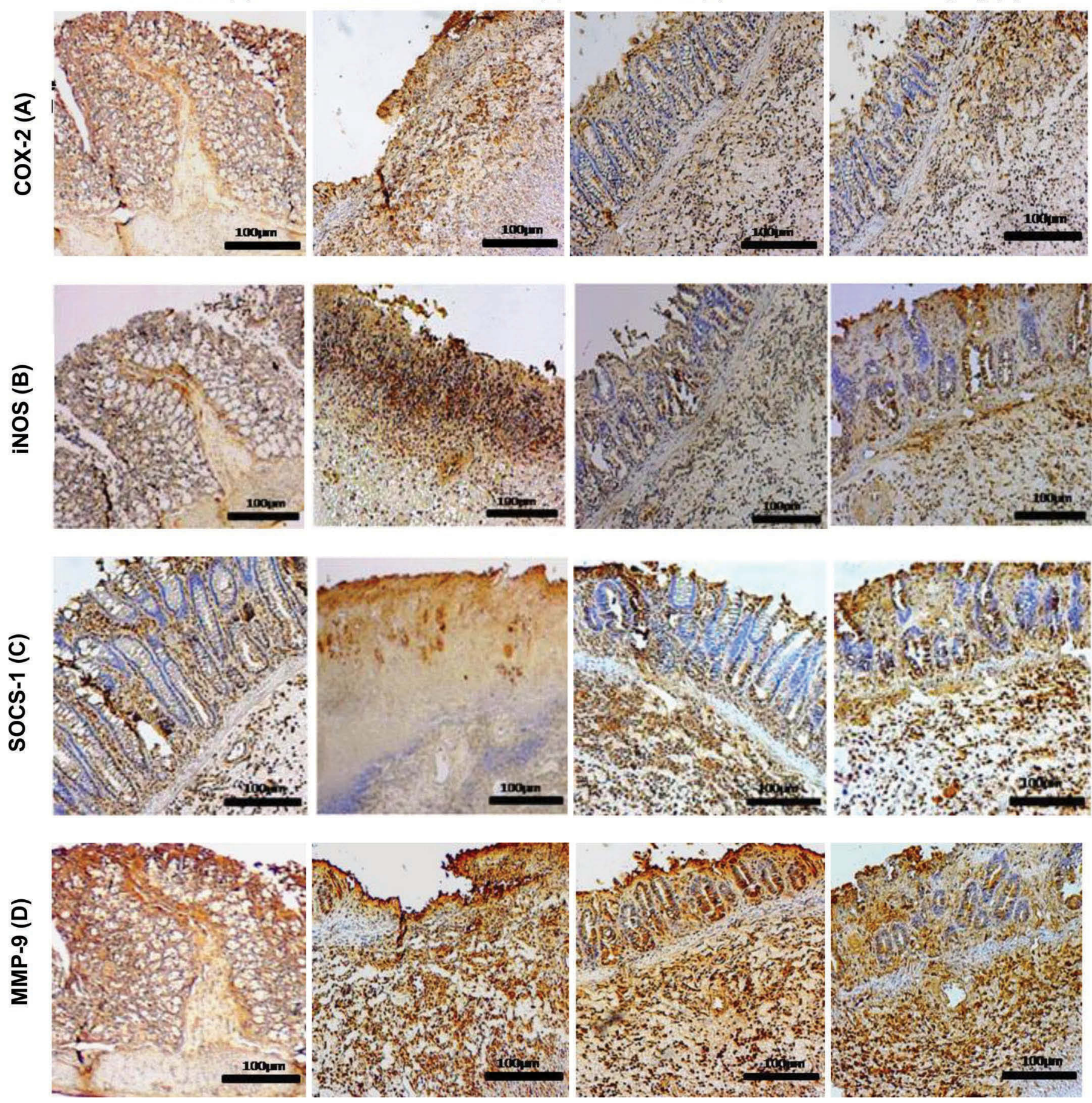

Figure 5. Immunohistochemical analysis of the colonic tissue in the acetic acid-induced colitis rats. Immunoexpression for cyclooxygenase (COX)-2 (A), inducible nitric oxide synthase (iNOS; B), suppressor of cytokine signaling-1 (SOCS-1; C), and matrix metalloproteinase (MMP)9 (D). For each antigen, 3 immunostained sections were examined per animal $(\mathrm{n}=5)$. 40× magnification, scale bar $=100 \mu$ m; 1 : noncolitic; 2 : acetic acid control; 3: sulfasalazine (SAZ); 4: goat whey (GW). Color version available online. 
protective effects observed in the treatments against the altered oxidative status that occurs in the inflamed colonic tissue, as previously reported (Witaicenis et al., 2012; Pearl et al., 2013).

In fact, an amelioration in the colonic oxidative stress was achieved after treating colitic rats with GW or SAZ, as seen by their ability to increase the colonic content of the antioxidant peptide glutathione and to decrease MDA production. A decrease in MDA production is a marker of lipid peroxidation, a process that typically occurs after a release of free radicals in inflammatory conditions (Zhang et al., 2009).

The important pathogenic role that neutrophil infiltrates in the lamina propria play in patients suffering from IBD has also been well documented. This can contribute to tissue damage through the release of inflammatory mediators, including reactive oxygen species and proteases, as well as proinflammatory cytokines such as TNF- $\alpha$ and IL-1 (Ardizzone and Porro, 2002; Fournier and Parkos, 2012).

Our study revealed that GW and SAZ were able to inhibit the synthesis of these proinflammatory cytokines in colitic rats, which contributes to their significant anti-inflammatory effect. Both GW and SAZ have been described as playing a key role in the development and maintenance of the inflammatory response in the intestine (Sarra et al., 2010). Different mechanisms can be involved in the reduction of the neutrophil infiltration observed in the treated colitic rats, and one of these may result from the inhibitory effect exerted by the treatments on $\mathrm{LTB}_{4}$ production, an eicosanoid with potent chemotactic properties that has been described to be a pathogenic mediator in intestinal inflammation, as its synthesis blockade has been reported to be beneficial in experimental colitis (Gálvez et al., 2001).

Furthermore, and similar to that previously reported in other experimental models of rodent colitis (Algieri et al., 2014), the intestinal inflammatory process is associated with increased expression of inducible enzymes, such as iNOS and COX-2, which contribute to produce mediators that have deleterious effects on the intestinal mucosa. Thus, the inhibition in the expression of COX-2 could account for the improvement of the inflammatory process, given its pathogenic role in intestinal inflammation, after their stimulation by luminal pathogens through toll-like receptors (Sheibanie et al., 2007). In fact, its downregulation has been proposed to be involved in the mechanism of action of drugs currently used in human IBD, including aminosalicylates (Eliakim et al., 1992). In addition to COX-2, iNOS is also largely expressed in inflammatory conditions, being responsible for nitric oxide overproduction that may be harmful to the colon integrity and function, thus collaborating in the progress of the intestinal damage (Grisham et al., 2002).

In addition, the acetic acid-induced colitis was also associated with an increased expression of the MMP-9, a family of zinc-containing proteases that was reduced after GW treatment. Certainly, the increased expression of colonic MMP-9 may lead to an accelerated degradation of the matrix in inflammatory intestinal conditions, and the inhibition of this enzyme expression has been associated with the intestinal anti-inflammatory effects. Additionally, it has been reported that MMP can activate a wide range of cytokines, chemokines, receptors, adhesion molecules, and signaling molecules, thus clearly contributing to inflammation in these intestinal conditions (O'Sullivan et al., 2015).

Finally, SOCS-1 has been described to downregulate the cytokine-mediated signaling, mainly that which is activated by TLR signaling-induced cytokines (Fujimoto and Naka, 2010). Colonic inflammation was associated with decreased expression of this protein in comparison with the noncolitic group, whereas this expression increased in both treated groups (GW and SAZ), thus promoting its suppressing role on proinflammatory cytokines and restoring the altered immune responses that ameliorates colonic inflammation induced by acetic acid in rats. This was similar to that described in other models of murine colitis (Chinen et al., 2006).

Previous studies have reported the immunomodulatory effects of oligosaccharides from GW through reduction of leukocyte adhesion to the endothelial wall (Thum et al., 2015). Also, due to the similarity of human milk oligosaccharides to epithelial cell surface carbohydrates, their ability to inhibit the adhesion of pathogens to the cell surface in the intestinal mucosa has been reported (Kunz and Rudloff, 2008). All these actions can contribute to the beneficial effects ascribed to these food compounds in experimental intestinal inflammation in mice (Daddaoua et al., 2006; LaraVilloslada et al., 2006) and can justify the beneficial effects of GW demonstrated in the present study.

In summary, our data confirm that the administration of GW exerts an intestinal preventive effect in the colitis acetic acid rat model. This effect was associated with an amelioration of the altered immune response that characterizes intestinal inflammation. These results suggest that GW may have an important role in preventing intestinal inflammation. However, further studies are needed to gain more knowledge about the mechanisms involved in the beneficial effects of these compounds in intestinal function and their implication on human intestinal inflammation. 


\section{ACKNOWLEDGMENTS}

The authors are grateful to Conselho Nacional de Desenvolvimento Científico e Tecnológico (CNPq, Brazil; process: 400738/2013-9 MEC/MCTI/CNPq/ FAPs no 71/2013 - PVE/ line 2, process: 473907/2012-7 MCT/ CNPq/ Universal nº14/2012 and process: 403020/2013$1 \mathrm{MCTI} / \mathrm{CT}-\mathrm{AGRO} / \mathrm{CNPq} / \mathrm{n}^{\circ} 39 / 2013$ Line 2) for the financial support for the development of this research, Laboratory Food Chemistry (Universidade Federal da Paraiba, Brazil) for drying goat whey, and to the Junta de Andalucia (AGR-6826 and CTS 164). The Centro de Investigación Biomédica en Enfermedades Hepáticas y Digestivas (CIBER-EHD) is funded by the Instituto de Salud Carlos III (Granada, Spain).

\section{REFERENCES}

Agarwal, S., J. Deschner, P. Long, A. Verma, C. Hofman, C. H. Evans, and N. Piesco. 2004. Role of NF-kappaB transcription factors in antiinflammatory and proinflammatory actions of mechanical signals. Arthritis Rheum. 50:3541-3548. http://dx.doi.org/10.1002/ art.20601.

Ahmed, A. S., T. El-Bassiony, L. M. Elmalt, and H. R. Ibrahim. 2015. Identification of potent antioxidant bioactive peptides from goat milk proteins. Food Res. Int. 74:80-88. http://dx.doi. $\operatorname{org} / 10.1016 /$ j.foodres.2015.04.032.

Albrecht, S., J. A. Lane, K. M. K. A. Busadah, S. D. Carrington, R. M. Hickey, and P. M. Rudd. 2014. A comparative study of free oligosaccharides in the milk of domestic animals. Br. J. Nutr. 111:1313-1328. http://dx.doi.org/10.1017/S0007114513003772.

Algieri, F., A. Rodriguez-Nogales, N. Garrido-Mesa, P. Zorrilla, N. Burkard, I. Pischel, H. Sievers, B. Benedek, B. Feistel, B. Walbroel, M. E. Rodriguez-Cabezas, and J. Galvez. 2014. Intestinal antiinflammatory activity of the Serpylli herba extract in experimental models of rodent colitis. J. Crohns Colitis 8:775-788. http:// dx.doi.org/10.1016/j.crohns.2013.12.012.

Anderson, M. E. 1985. Determination of glutathione and glutathione disulfide in biological samples. Methods Enzymol. 113:548-555.

AOAC International. 2005. Official Methods of Analysis. 18th ed. AOAC International, Rockville, MD.

Ardizzone, S., and G. B. Porro. 2002. Inflammatory bowel disease: New insights into pathogenesis and treatment. J. Intern. Med. 252:475-496.

Ardizzone, S., and G. B. Porro. 2005. Biologic therapy for inflammatory bowel disease. Drugs 65:2253-2286.

Bell, C. J., D. G. Gall, and J. L. Wallace. 1995. Disruption of colonic electrolyte transport in experimental colitis. Am. J. Physiol. 268:G622-630.

Bernstein, C. N. 2015. Treatment of IBD: Where we are and where we are going. Am. J. Gastroenterol. 110:114-126.

Bressler, B., J. K. Marshall, C. N. Bernstein, A. Bitton, J. Jones, G. I. Leontiadis, R. Panaccione, A. H. Steinhart, F. Tse, and B. Feagan. 2015. Clinical practice guidelines for the medical management of nonhospitalized ulcerative colitis: The Toronto consensus on behalf of the Toronto Ulcerative Colitis Consensus Group. Gastroenterology 148:1035-1058.e3. http://dx.doi.org/10.1053/j. gastro.2015.03.001.

Chinen, T., T. Kobayashi, H. Ogata, G. Takaesu, H. Takaki, M. Hashimoto, H. Yagita, H. Nawata, and A. Yoshimura. 2006. Suppressor of cytokine signaling-1 regulates inflammatory bowel disease in which both IFN gamma and IL-4 are involved. Gastroenterology 130:373-388. http://dx.doi.org/10.1053/j.gastro.2005.10.051.

Daddaoua, A., V. Puerta, A. Zarzuelo, M. D. Suárez, F. Sánchez de Medina, and O. Martínez-Augustin. 2006. Goat milk oligosaccha- rides are anti-inflammatory in rats with hapten-induced colitis. J. Nutr. 136:672-676.

Dedon, P. C., and S. R. Tannenbaum. 2004. Reactive nitrogen species in the chemical biology of inflammation. Arch. Biochem. Biophys. 423:12-22. http://dx.doi.org/10.1016/j.abb.2003.12.017.

Eliakim, R., F. Karmeli, M. Chorev, E. Okon, and D. Rachmilewitz. 1992. Effect of drugs on colonic eicosanoid accumulation in active ulcerative colitis. Scand. J. Gastroenterol. 27:968-972.

Esterbauer, H., and K. H. Cheeseman. 1990. Determination of aldehydic lipid peroxidation products: Malonyldialdihyde and 4-hydroxynonenal. Methods Enzymol. 186:407-421.

Fournier, B. M., and C. A. Parkos. 2012. The role of neutrophils during intestinal inflammation. Mucosal Immunol. 5:354-366. http:// dx.doi.org/10.1038/mi.2012.24.

Fujimoto, M., and T. Naka. 2010. SOCS1, a negative regulator of cytokine signals and TLR responses, in human liver diseases. Gastroenterol. Res. Pract. 2010:1-7. http://dx.doi. org $/ 10.1155 / 2010 / 470468$.

Gálvez, J., G. Coelho, M. E. Crespo, T. Cruz, M. E. Rodriguez-Cabezas, A. Concha, M. Gonzalez, and A. Zarzuelo. 2001. Intestinal anti-infammatory activity of morin on chronic experimental colitis in the rat. Aliment. Pharmacol. Ther. 15:2027-2039.

Galvez, J., M. Comalada, and J. Xaus. 2010. Prebiotics and probiotics in experimental models of rodent colitis: Lessons in treatment or prevention of inflammatory bowel diseases. Pages 589-610 in Bioactive Foods in Promoting Health. R. R. Watson and V. R. Preedy, ed. Academic Press, Oxford, UK.

Grisham, M. B., K. P. Pavlick, F. S. Laroux, J. Hoffman, S. Bharwani, and R. E. Wolf. 2002. Nitric oxide and chronic gut inflammation: Controversies in inflammatory bowel disease. J. Investig. Med. 50:272-283.

Guerra, G. C. B., A. A. Araujo, G. A. Lira, M. N. Melo, K. K. O. Souto, D. Fernandes, A. L. Silva, and R. F. Araujo Junior. 2015. Telmisartan decreases inflammation by modulating TNF-a, IL-10, and RANK/RANKL in a rat model of ulcerative colitis. Pharmacol. Rep. 67:520-526. http://dx.doi.org/10.1016/j.pharep.2014.12.011.

Hernández-Ledesma, B., M. Ramos, and J. A. Gómez-Ruiz. 2011. Bioactive components of ovine and caprine cheese whey. Small Rumin. Res. 101:196-204. http://dx.doi.org/10.1016/j. smallrumres.2011.09.040.

Hur, S. J., S. H. Kang, H. S. Jung, S. C. Kim, H. S. Jeon, I. H. Kim, and J. D. Lee. 2012. Review of natural products actions on cytokines in inflammatory bowel disease. Nutr. Res. 32:801-816. http://dx.doi.org/10.1016/j.nutres.2012.09.013.

Hyun, J. G., and L. Mayer. 2006. Mechanisms underlying inflammatory bowel disease. Drug Discov. Today 3:457-462. http://dx.doi. org/10.1016/j.ddmec.2006.11.010.

Kolios, G., V. Valatas, and S. G. Ward. 2004. Nitric oxide in inflammatory bowel disease: A universal messeger in an unsolved puzzle. Immunology 113:427-437. http://dx.doi.org/10.1111/j.13652567.2004.01984.x.

Krawisz, J. E., P. Sharon, and W. F. Stenson. 1984. Quantitative assay for acute intestinal inflammation in rat and hamster models. Gastroenterology 87:1344-1350.

Kunz, C., and S. Rudloff. 2008. Potential anti-inflammatory and antiinfectious effects of human milk oligosaccharides. Adv. Exp. Med. Biol. 606:455-465.

Kunz, C., S. Rudloff, W. Baier, N. Klein, and S. Strobel. 2000. Oligosaccharides in human milk: structural, functional, and metabolic aspects. Annu. Rev. Nutr. 20:699-722.

Lara-Villoslada, F., E. Debras, A. Nieto, A. Concha, J. Galvez, E. Lopez-Huertas, J. Boza, C. Obled, and J. Xaus. 2006. Oligosaccharides isolated from goat milk reduce intestinal inflammation in a rat model of dextran sodium sulfate-induced colitis. Clin. Nutr. 25:477-488.

MacPherson, B. R., and C. J. Pfeiffer. 1978. Experimental production of diffuse colitis in rats. Digestion 17:135-150.

Magalhães, J., F. D. Castro, P. B. Carvalho, J. F. Machado, S. Leite, M. J. Moreira, and J. Cotter. 2015. Disability in inflammatory bowel disease: Translation to Portuguese and validation of the 
"Inflammatory Bowel Disease - Disability Score". GE Port. J. Gastroenterol. 22:4-14. http://dx.doi.org/10.1016/j.jpge.2014.10.002.

Martinez-Ferez, A., S. Rudolff, A. Guadix, C. A. Henkel, G. Pohlentz, J. J. Boza, E. M. Guadix, and C. Kunz. 2006. Goat's milk as a natural source of lactose-derived oligosaccharides: isolation by membrane technology. Int. Dairy J. 16:173-181.

Millar, A. D., D. S. Rampton, C. L. Chander, A. W. Claxson, A. Blades, A. Coumbe, J. Panetta, C. J. Morris, and D. R. Blake. 1996. Evaluating the antioxidant potential of new treatments for inflammatory bowel disease using a rat model of colitis. Gut $39: 407-415$.

Molodecky, N. A., I. S. Soon, D. M. Rabi, W. A. Ghali, M. Ferris, G. Chernoff, E. I. Benchimol, R. Panaccione, S. Ghosh, H. W. Barkema, and G. G. Kaplan. 2012. Increasing Incidence and Prevalence of the Inflammatory Bowel Diseases With Time, Based on Systematic Review. Gastroenterology 142:46-54.e42. http://dx.doi. org/10.1053/j.gastro.2011.10.001.

NRC. 1985. Guide for the Care and Use of Laboratory Animals. Natl. Acad. Press, Washington, DC.

NRC. 2007. Nutrient Requirements of Small Ruminants: Sheep, Goats, Cervids, and New World Camelids. Natl. Acad. Press, Washington, DC.

Naugler, W. E., and M. Karin. 2008. NF-KappaB and cancer-identifying targets and mechanisms. Curr. Opin. Genet. Dev. 18:19-26. http://dx.doi.org/10.1016/j.gde.2008.01.020.

Nørgård, B. M., J. Nielsen, K. Fonager, J. Kjeldsen, B. A. Jacobsen, and N. Qvist. 2014. The incidence of ulcerative colitis (1995-2011) and Crohn's disease (1995-2012) - Based on nationwide Danish registry data. J. Crohns Colitis 8:1274-1280.

O'Sullivan, S., J. F. Gilmer, and C. Medina. 2015. Matrix metalloproteinases in inflammatory bowel disease: An update. Mediat. Inflamm. 2015:964131. http://dx.doi.org/10.1155/2015/964131.

Oliveira, M. E. G., E. F. Garcia, R. C. R. E. Queiroga, and E. L. Souza. 2012. Technological, physicochemical and sensory characteristics of a Brazilian semi-hard goat cheese (coalho) with added probiotic lactic acid bacteria. Sci. Agric. 69:370-379.

Pearl, D. S., K. Shah, M. A. Whittaker, H. Nitch-Smith, J. F. Brown, J. K. Shute, and T. M. Trebble. 2013. Cytokine mucosal expression in ulcerative colitis, the relationship between cytokine release and disease activity. J. Crohns Colitis 7:481-489. http://dx.doi. org/10.1016/j.crohns.2012.07.022

Roberfroid, M., G. R. Gibson, L. Hoyles, A. L. McCartney, R. Rastall, I. Rowland, D. Wolvers, B. Watzl, H. Szajewska, B. Stahl, F. Guarner, F. Respondek, K. Whelan, V. Coxam, M. J. Davicco, L. Léotoing, Y. Wittrant, N. M. Delzenne, P. D. Cani, A. M. Neyrinck, and A. Meheust. 2010. Prebiotic effects: metabolic and health benefits. Br. J. Nutr. 104(Suppl 2):S1-S63.

Rouzer, C. A., and L. J. Marnett. 2009. Cyclooxygenases: Structural and functional insights. J. Lipid Res. 50:S29-S34. http://dx.doi. org/10.1194/jlr.R800042-JLR200.

Sakthivel, K. M., and C. Guruvayoorappan. 2013. Amentoflavone inhibits iNOS, COX-2 expression and modulates cytokine profile,
NF- $\kappa \mathrm{B}$ signal transduction pathways in rats with ulcerative colitis Int. Immunopharmacol. 17:907-916. http://dx.doi.org/10.1016/j. intimp.2013.09.022

Salas, A., M. Gironella, A. Salas, A. Soriano, M. Sans, J. Iovanna, J. M. Pique, and J. Panes. 2002. Nitric oxide supplementation ameliorates dextran sulfate sodium-induced colitis in mice. Lab. Invest. 82:597-607.

Sang, L., B. Chang, C. Dai, N. Gao, W. Liu, and M. Jiang. 2014 Heat-killed VSL\#3 ameliorates dextran sufate sodium (Dss)-induced acute experimental colitis in rats. Int. J. Mol. Sci. 15:15-28. http://dx.doi.org/10.3390/ijms15010015.

Sarra, M., F. Pallone, T. T. Macdonald, and G. Monteleone. 2010. IL23/IL-17 axis in IBD. Inflamm. Bowel Dis. 16:1808-1813.

Sheibanie, A. F., J. H. Yen, T. Khayrullina, F. Emig, M. Zhang, R. Tuma, and D. Ganea. 2007. The proinflammatory effect of prostaglandin E2 in experimental inflammatory bowel disease is mediated through the IL-23/IL-17 axis. J. Immunol. 178:8138-8147.

Siegel, C. A. 2011. Review article: explaining risks of inflammatory bowel disease therapy to patients. Aliment. Pharmacol. Ther. 33:23-32.

Sousa, Y. R. F., M. A. S. Vasconcelos, R. G. Costa, C. A. A. Azevedo Filho, E. P. Paiva, and R. C. R. E. Queiroga. 2015. Sialic acid content of goat milk during lactation. Livest. Sci. 177:175-180. http:// dx.doi.org/10.1016/j.livsci.2015.04.005.

Strober, W., I. Fuss, and P. Mannon. 2007. The fundamental basis of inflammatory bowel disease. J. Clin. Invest. 117:514-521. http:// dx.doi.org/10.1172/JCI30587.

Strober, W., and I. J. Fuss. 2011. Proinflammatory cytokines in the pathogenesis of inflammatory bowel diseases. Gastroenterology 140:1756-1767. http://dx.doi.org/10.1053/j.gastro.2011.02.016.

Thum, C., A. Cookson, W. C. McNabb, N. C. Roy, and D. Otter. 2015 Composition and enrichment of caprine milk oligosaccharides from New Zealand Saanen goat cheese whey. J. Food Compos. Anal 42:30-37. http://dx.doi.org/10.1016/j.jfca.2015.01.022.

Witaicenis, A., A. C. Luchini, C. A. Hiruma-Lima, S. L. Felisbino, N. Garrido-Mesa, P. Utrilla, J. Gálvez, and L. C. Di Stasi. 2012. Suppression of TNBS-induced colitis in rats by 4-methylesculetin, a natural coumarin: Comparison with prednisolone and sulphasalazine. Chem-Biol. Interac. 195:76-85. http://dx.doi.org/10.1016/j. cbi.2011.11.004.

Zea-Iriarte, W. L., K. Makiyama, S. Goto, K. Murase, Y. Urata, I. Sekine, K. Hara, and T. Kondo. 1996. Impairment of antioxidants in colonic epithelial cells isolated from trinitrobenzene sulphonic acid-induced colitis rats-Protective effect of rebamipide. Scand J. Gastroenterol. 31:985-992.

Zhang, D. K., L. N. Cheng, X. L. Huang, W. Shi, J. Y. Xiang, and H. T. Gan. 2009. Tetrandrine ameliorates dextran-sulfate-sodium-induced colitis in mice through inhibition of nuclear factorkappaB activation. Int. J. Colorectal Dis. 24:5-12. http://dx.doi. org/10.1007/s00384-008-0544-7. 